This item was submitted to Loughborough's Institutional Repository (https://dspace.lboro.ac.uk/) by the author and is made available under the following Creative Commons Licence conditions.

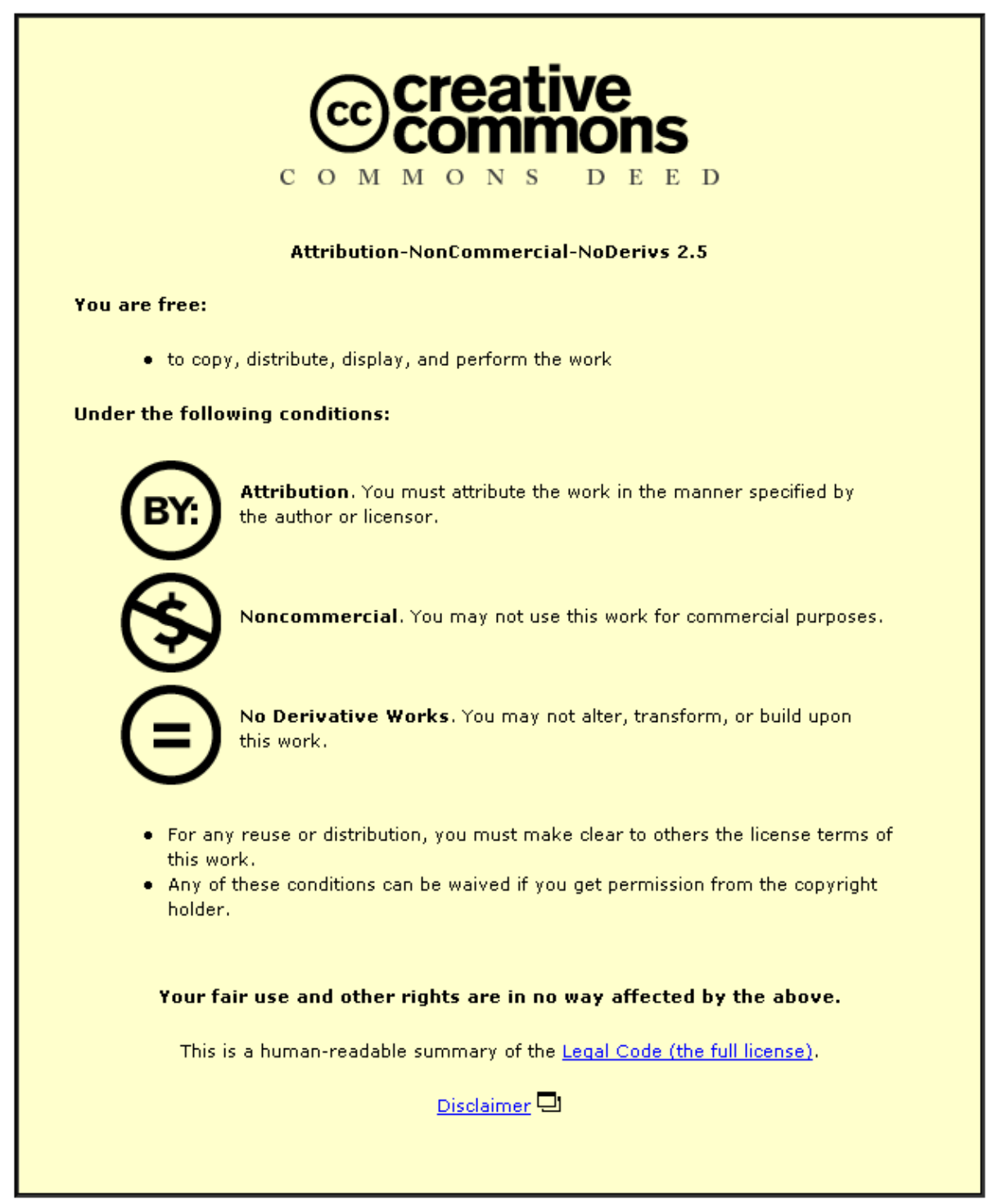

For the full text of this licence, please go to: http://creativecommons.org/licenses/by-nc-nd/2.5/ 


\title{
Multiuser Orthogonal Space-Division Multiplexing with Iterative Water-Filling Algorithm
}

\author{
Zhilan Xiong, Ranaji Krishna, Sangarapillai Lambotharan and Jonathon A. Chambers \\ Advanced Signal Processing Group \\ Loughborough University \\ Leicestershire, UK, LE11 3TU \\ Email: \{Z.Xiong, R.Krishna, S.Lambotharan, J.A.Chambers\}@lboro.ac.uk
}

\begin{abstract}
The problem of multiuser multiplexing with a MIMO sub system for each individual user is considered. We demonstrate that the capacity performance of the null space based spatial multiplexing schemes can be improved with iterative power allocation within the iterative design process. We considered water-filling based local and global power allocation and demonstrate that both schemes outperform the existing null space based spatial diversity technique in terms of mean capacity and outage capacity.

Index Terms-Channel capacity, multiple-input multipleoutput (MIMO), power allocation, spatial diversity techniques
\end{abstract}

\section{INTRODUCTION}

Multiuser wireless communication systems employing multiple antennas at both the transmitter and the receiver have attracted much interest recently due to their potential to enhance significantly capacity [1], [2]. When the basestation (BS) and multiuser terminals employ multiple antennas, a multipleinput multiple-output (MIMO) channel can be formed between each user terminal and BS. Since in the multiuser downlink MIMO system, the BS will communicate with several users simultaneously over the same frequency band [3], inter-user interference (IUI) becomes a major obstacle limiting the radio link layer performance [4]. If the IUI is not mitigated carefully, the sum capacity of the multiuser MIMO system will severely degrade [5]-[7].

In this paper, we propose a multiuser multistream MIMO downlink system based on iterative water-filling and a null space method. Due to the presence of IUI, the power allocation of each user influences not only its own performance, but also the performances of other users [8]. Hence, we use a two-stage iterative approach. The IUI is first cancelled by employing orthogonal space-division multiplexing (OSDM) [9], and then a water-filling algorithm is used to allocate power for each stream and each user.

In the OSDM scheme proposed in [9], the multiuser signals are projected onto orthogonal subspaces to eliminate IUI. Then the singular value decomposition (SVD) is performed to decompose the equivalent MIMO channel of each user into a set of single-input single-output (SISO) subchannels. The

This work has been supported by the Engineering and Physical Sciences Research Council of the UK under the grant, EPSRC E041817. work in [9], considered an iterative approach to design the set of beamformers required for multiuser MIMO systems. According to the design, all the subchannels have been assumed to be used for data transmission. However, for optimizing capacity, different powers need to be allocated for each of the subchannels using a water-filling approach. This may result in a number of the weaker subchannels being discarded from the data transmission. Such selective removal of subchannels, during the iterative design process, could provide additional degrees of freedom to mitigate IUI. Therefore, we propose to introduce water-filling based power allocation and subchannel selection to the work in [9] so that the proposed design provides more flexibility for controlling IUI, in addition to maximizing the sum capacity.

We propose two kinds of power allocation schemes: local power allocation and global power allocation. Local power allocation means that the power allocated for each user is limited individually. Global power allocation means that the sum of the transmit power at the BS is restricted, and the power is allocated jointly to all users and all data streams.

The rest of the paper is organized as follows. A multiuser multistream MIMO system is described in the next section. After the introduction of the OSDM algorithm in Section III, two different OSDM schemes with power allocation are proposed in Section IV. Section V presents the simulation results, and conclusion are drawn in Section VI.

\section{SySTEM MODEL}

Consider a downlink communication system with a BS communicating with $K$ users. There are $N_{t}$ antennas at the BS and $N_{r, i}$ receiver antennas for the $i$ th user. Let $\mathbb{C}$ denote the set of complex numbers and $\mathbf{s}_{i} \in \mathbb{C}^{N_{m, i} \times 1}$ denote the transmitted data symbol vector intended for user $i$, where $N_{m, i}$ denotes the number of data streams transmitted simultaneously for user $i$ such that

$$
\mathbf{s}_{i}=\left[\begin{array}{llll}
s_{i, 1} & s_{i, 2} & \cdots & s_{i, N_{m, i}}
\end{array}\right]^{T}
$$

where the superscript $T$ denotes vector transpose. Let $p_{i, j}$ denote the power allocated to element $s_{i, j}$, and define a vector $\tilde{\mathbf{s}}_{i}$ as

$$
\tilde{\mathbf{s}}_{i}=\left[\begin{array}{llll}
\sqrt{p_{i, 1}} s_{i, 1} & \sqrt{p_{i, 2}} s_{i, 2} & \cdots & \sqrt{p_{i, N_{m, i}}} s_{i, N_{m, i}}
\end{array}\right]^{T}
$$


Before being transmitted over the channel, this symbol vector is multiplied by a precoding matrix $\mathbf{W}_{i} \in \mathbb{C}^{N_{t} \times N_{m, i}}$. Hence, the overall transmitted signal vector $\mathbf{x} \in \mathbb{C}^{N_{t} \times 1}$ can be written as

$$
\mathbf{x}=\sum_{k=1}^{K} \mathbf{W}_{k} \tilde{\mathbf{s}}_{k}
$$

The input-output relationship of the channel for the $i$ th user can be written as

$$
\mathbf{y}_{i}=\mathbf{H}_{i} \mathbf{x}+\mathbf{n}_{i}
$$

where $\mathbf{y}_{i} \in \mathbb{C}^{N_{r, i} \times 1}$ denotes the received signal vector by the antennas of user $i ; \mathbf{H}_{i} \in \mathbb{C}^{N_{r, i} \times N_{t}}$ denotes the channel matrix from the transmit antennas to the receive antennas of user $i ; \mathbf{n}_{i} \in \mathbb{C}^{N_{r, i} \times 1}$ denotes the noise vector, whose elements are zero-mean circularly symmetric Gaussian random variables with variance $\sigma_{i}^{2}$, and $E\left\{\mathbf{n}_{i} \mathbf{n}_{i}^{H}\right\}=\sigma_{i}^{2} \mathbf{I}_{N_{r, i}}$, where $\mathbf{I}_{N_{r, i}}$ is an $N_{r, i} \times N_{r, i}$ identity matrix and the superscript $H$ denotes conjugate transpose.

\section{Multiuser OSDM Method}

A multiuser OSDM technique for a downlink multiuser multistream MIMO channel has been proposed in [9]. The method is based on projecting the multiuser signals onto orthogonal subspaces to eliminate IUI, and then performing an SVD to decompose the resulting MIMO channel into a set of SISO subchannels.

Suppose, in the receiver, the received signal $\mathbf{y}_{i}$ is multiplied by a matrix $\mathbf{T}_{i}$, i.e.,

$$
\mathbf{y}_{i}^{\prime}=\mathbf{T}_{i} \mathbf{y}_{i}
$$

where vector $\mathbf{y}_{i}^{\prime} \in \mathbb{C}^{N_{m, i} \times 1}$ and matrix $\mathbf{T}_{i} \in \mathbb{C}^{N_{m, i} \times N_{r, i}}$.

Substituting (3) and (4) into (5), and collecting the received signals of all users into a single vector, we obtain

$$
\begin{aligned}
{\left[\begin{array}{c}
\mathbf{y}_{1}^{\prime} \\
\mathbf{y}_{2}^{\prime} \\
\vdots \\
\mathbf{y}_{K}^{\prime}
\end{array}\right]=} & {\left[\begin{array}{cccc}
\mathbf{T}_{1} \mathbf{H}_{1} \mathbf{W}_{1} & \mathbf{T}_{1} \mathbf{H}_{1} \mathbf{W}_{2} & \ldots & \mathbf{T}_{1} \mathbf{H}_{1} \mathbf{W}_{K} \\
\mathbf{T}_{2} \mathbf{H}_{2} \mathbf{W}_{1} & \mathbf{T}_{2} \mathbf{H}_{2} \mathbf{W}_{2} & \ldots & \mathbf{T}_{2} \mathbf{H}_{2} \mathbf{W}_{K} \\
\vdots & \vdots & \ddots & \vdots \\
\mathbf{T}_{K} \mathbf{H}_{K} \mathbf{W}_{1} & \mathbf{T}_{K} \mathbf{H}_{K} \mathbf{W}_{2} & \ldots & \mathbf{T}_{K} \mathbf{H}_{K} \mathbf{W}_{K}
\end{array}\right] } \\
& {\left[\begin{array}{c}
\tilde{\mathbf{s}}_{1} \\
\tilde{\mathbf{s}}_{2} \\
\vdots \\
\tilde{\mathbf{s}}_{K}
\end{array}\right]+\left[\begin{array}{c}
\mathbf{n}_{1}^{\prime} \\
\mathbf{n}_{2}^{\prime} \\
\vdots \\
\mathbf{n}_{K}^{\prime}
\end{array}\right] }
\end{aligned}
$$

where $\mathbf{n}_{i}^{\prime}=\mathbf{T}_{i} \mathbf{n}_{i}$.

The underlying principle in the OSDM method is to eliminate IUI by making $\mathbf{T}_{i} \mathbf{H}_{i} \mathbf{W}_{j}$ equal to a zero matrix for $i \neq j$, i.e.,

$$
\mathbf{W}_{i} \in \operatorname{null}\left\{\tilde{\mathbf{H}}_{i}^{e}\right\}
$$

where

$$
\tilde{\mathbf{H}}_{i}^{e}=\left[\begin{array}{c}
\mathbf{T}_{1} \mathbf{H}_{1} \\
\vdots \\
\mathbf{T}_{i-1} \mathbf{H}_{i-1} \\
\mathbf{T}_{i+1} \mathbf{H}_{i+1} \\
\vdots \\
\mathbf{T}_{K} \mathbf{H}_{K}
\end{array}\right] \in \mathbb{C}^{\left(\sum_{k=1, k \neq i}^{K} N_{m, k}\right) \times N_{t}}
$$

In this case, $\mathbf{y}_{i}^{\prime}$ can be simplified as

$$
\mathbf{y}_{i}^{\prime}=\mathbf{T}_{i} \mathbf{H}_{i} \mathbf{W}_{i} \tilde{\mathbf{s}}_{i}+\mathbf{n}_{i}^{\prime}
$$

Let $\mathbf{G}_{i} \in \mathbb{C}^{N_{t} \times\left(N_{t}-\sum_{k=1, k \neq i}^{K} N_{m, k}\right)}$ denote the orthonormal basis of null $\left\{\tilde{\mathbf{H}}_{i}^{e}\right\}$. Decomposing $\mathbf{H}_{i} \mathbf{G}_{i}$ with the SVD, we obtain

$$
\mathbf{H}_{i} \mathbf{G}_{i}=\mathbf{U}_{i} \boldsymbol{\Lambda}_{i} \mathbf{V}_{i}^{H}
$$

where $\mathbf{U}_{i} \in \mathbb{C}^{N_{r, i} \times N_{r, i}}, \boldsymbol{\Lambda}_{i} \in \mathbb{C}^{N_{r, i} \times\left(N_{t}-\sum_{k=1, k \neq i}^{K} N_{m, k}\right)}$ and $\mathbf{V}_{i} \in \mathbb{C}^{\left(N_{t}-\sum_{k=1, k \neq i}^{K} N_{m, k}\right) \times\left(N_{t}-\sum_{k=1, k \neq i}^{K} N_{m, k}\right)}$. Let $\tilde{\mathbf{U}}_{i}=\left.\mathbf{U}_{i}\right|_{1 \leftrightarrow N_{m, i}}$ and $\tilde{\mathbf{V}}_{i}=\left.\mathbf{V}_{i}\right|_{1 \leftrightarrow N_{m, i}}$, where the notation $\left.\cdot\right|_{1 \leftrightarrow N_{m, i}}$ denotes collecting the column vectors that correspond to the $N_{m, i}$ largest singular values. Let $\tilde{\Lambda}_{i}$ be a diagonal matrix, whose diagonal elements are the $N_{m, i}$ largest singular values of $\mathbf{H}_{i} \mathbf{G}_{i}$, i.e., $\lambda_{i, 1}, \lambda_{i, 2}, \ldots, \lambda_{i, N_{m, i}}$.

The weight matrices at the transmitter and the receiver are chosen as

$$
\mathbf{W}_{i}=\mathbf{G}_{i} \tilde{\mathbf{V}}_{i}
$$

and

$$
\mathbf{T}_{i}=\tilde{\mathbf{U}}_{i}^{H}
$$

i.e. the signal for the $i$ th user is transmitted solely through the null space of $\tilde{\mathbf{H}}_{i}^{e}$, but the subspace of this null space is chosen as the one that maximises the projection onto the signal space of $\mathbf{H}_{i}$.

Substituting (10) and (11) into (8), yields

$$
\mathbf{y}_{i}^{\prime}=\tilde{\Lambda}_{i} \tilde{\mathbf{s}}_{i}+\mathbf{n}_{i}^{\prime}
$$

Using the above solutions, the iterative OSDM method has been formulated in [9] as follows.

1) Initialize $\mathbf{T}_{i}, \forall i$, as

$$
\mathbf{T}_{i}=\left[\begin{array}{ll}
\mathbf{I}_{N_{m, i} \times N_{r, i}} & \mathbf{0}_{N_{m, i} \times\left(N_{r, i}-N_{m, i}\right)}
\end{array}\right]
$$

where $N_{m, i} \leqslant N_{r, i}$.

2) For each user, calculate the orthonormal basis of $\operatorname{null}\left\{\tilde{\mathbf{H}}_{i}^{e}\right\}, \mathbf{G}_{i}$. Use the SVD of $\mathbf{H}_{i} \mathbf{G}_{i}$ to obtain $\tilde{\mathbf{U}}_{i}, \tilde{\boldsymbol{\Lambda}}_{i}$ and $\tilde{\mathbf{V}}_{i}$. Then $\mathbf{W}_{i}=\mathbf{G}_{i} \tilde{\mathbf{V}}_{i}$ and $\mathbf{T}_{i}=\tilde{\mathbf{U}}_{i}^{H}$ are used to update the matrices $\mathbf{W}_{i}$ and $\mathbf{T}_{i}$.

3) An error metric is computed as

$$
\varepsilon=\sum_{i=1}^{K}\left\|\tilde{\mathbf{H}}_{i}^{e} \mathbf{W}_{\mathbf{i}}\right\|_{F}^{2}
$$

where $\|\cdot\|_{F}$ denotes the Frobenius norm. If $\varepsilon \leqslant \varepsilon_{0}\left(=10^{-12}\right.$ typically), go to Step 4); otherwise, go back to Step 2).

4) Obtain the optimal precoding and encoding matrices $\mathbf{W}_{i}$ and $\mathbf{T}_{i}$ using (10) and (11), respectively, and then make 
$\left\|\mathbf{W}_{i}^{(j)}\right\|^{2}=1$, where $\mathbf{W}_{i}^{(j)}$ is the $j$ th column of $\mathbf{W}_{i}$ to satisfy the power constraint.

\section{OSDM Method with Power Allocation}

In this section, two kinds of power control schemes with OSDM are considered for the downlink multiuser MIMO system: local power allocation and global power allocation. Local power allocation is to maximize the capacity of the subchannel for each user under the constraint that the total power for each user is limited. Different from the local power allocation, the global power allocation maximizes the capacity of all users jointly with a constraint on the total power available at the transmitter. It means that the global power allocation achieves the maximization of total capacity, while the local power allocation emphasizes on maximizing capacity for each user. The use of power allocation using a water-filling technique in this multiuser scenario has another advantage. The power allocation may result in some of the weaker channels being discarded from data transmission. Therefore, the number of subchannels used for each user is reduced. This will provide more degrees of freedom for IUI cancellation during the iterative design procedure.

\section{A. Local Power Allocation}

As mentioned in Section III, the OSDM method can decompose the multiuser multistream channel into a set of single user MIMO channels. Having performed OSDM, the sub-MIMO system for each user is also decomposed into a set of SISO subchannels. Then, the channel capacity for user $i$ can be written as

$$
C_{i}=\sum_{j=1}^{N_{m, i}} \log _{2}\left(1+\frac{\lambda_{i, j}^{2}}{\sigma_{i}^{2}} p_{i, j}\right)
$$

where $\lambda_{i, j}$ is the $j$ th diagonal element of $\tilde{\boldsymbol{\Lambda}}_{i}$. So, according to the water-filling method, the optimal power allocation for data stream $s_{i, j}$ is given by

$$
p_{i, j}^{o p t}=\left(\mu-\frac{\sigma_{i}^{2}}{\lambda_{i, j}^{2}}\right)_{+}
$$

where $(x)_{+}$implies

$$
(x)_{+}= \begin{cases}x & \text { if } x \geq 0 \\ 0 & \text { if } x<0\end{cases}
$$

and $\mu$ must be chosen such that

$$
\sum_{j=1}^{N_{m, i}} p_{i, j}^{o p t}=p_{i}^{\max }
$$

where $p_{i}^{\max }$ is the maximum allowed power of the data symbol vector intended for user $i$. Therefore, the problem formulation for the local power allocation can be described as

Problem 1: Determine the optimum power allocation for each data stream so as to maximize capacity for each user

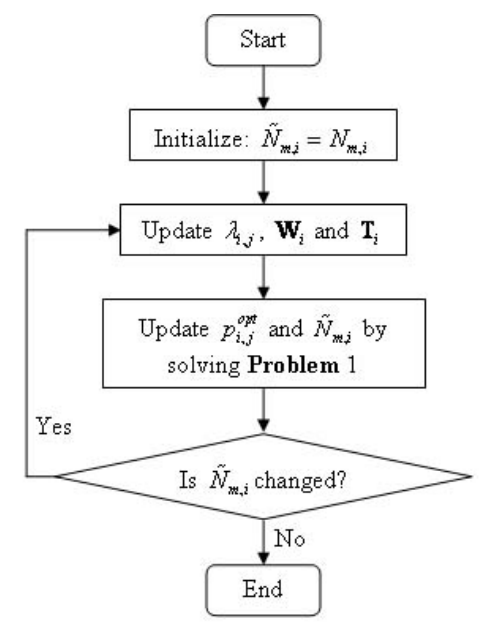

Fig. 1. Flow diagram representation of the local power allocation scheme.

under a per user power constraint, i.e.,

$$
\begin{array}{cl}
\max & \sum_{i=1}^{K} C_{i}=\sum_{i=1}^{K} \sum_{j=1}^{N_{m, i}} \log _{2}\left(1+\frac{\lambda_{i, j}^{2}}{\sigma_{i}^{2}} p_{i, j}\right) \\
\text { s.t. } & \sum_{j=1}^{N_{m, i}} p_{i, j}=p_{i}^{\max }, 1 \leq i \leq K \\
& p_{i, j} \geq 0,1 \leq i \leq K, 1 \leq j \leq N_{m, i}
\end{array}
$$

The water-filling algorithm is used to solve the above problem. The water-filling algorithm maximizes the subchannel capacity of each user by allocating power asymmetrically to each subchannel, so that a good channel obtains more power than a bad channel. Data streams with low signal-to-noise ratio (SNR) may not get any power. In this case, the number of active spatial subchannels is reduced. It means that the structure of the MIMO system is changed, and it is necessary to calculate the weight vectors and decompose iteratively the new MIMO channel into SISO subchannels by OSDM. Hence, if the allocated powers to some data streams are zero, then the channels excluding unused zero power channels should be decomposed again until all powers allocated to the data streams are larger than zero. Therefore, the OSDM with local power allocation is an iterative process, which can be described as follows:

1) Initialize: $\tilde{N}_{m, i}=N_{m, i}(i=1, \ldots, K)$, where $\tilde{N}_{m, i}$ is the number of data streams with nonzero power for user $i$, $N_{m, i}$ denotes the allowed maximum number of data streams for the $i$ th user.

2) Calculate $\lambda_{i, j}\left(j=1, \ldots, \tilde{N}_{m, i}\right), \mathbf{W}_{i}$ and $\mathbf{T}_{i}(i=$ $1, \ldots, K)$ using the iterative OSDM method.

3) Obtain the optimal power allocation $p_{i, j}^{o p t}(i=1, \ldots, K$, $j=1, \ldots, \tilde{N}_{m, i}$ ) to maximize the capacity of each user with individual power constraint using the water-filling algorithm, and set $p_{i, j}^{\text {opt }}=0\left(i=1, \ldots, K, j=\tilde{N}_{m, i}+1, \ldots, N_{m, i}\right)$.

4) Iterate steps 2) and 3) until no further reduction on the number of subchannels $\tilde{N}_{m, i}$ for every user is observed.

In this iterative process, as shown in Fig. $1, \tilde{N}_{m, i}$ is used to indicate if the optimal power allocation has been finished. Since $\tilde{N}_{m, i}$ is finite and monotonically decreased, the procedure is convergent. 


\section{B. Global Power Allocation}

The objective of the global power allocation is to maximize the capacity of all users combined under a total power constraint, as stated below,

Problem 2: Determine the optimum power allocation for each data stream and each user to maximize total capacity under a constraint on the total power available at the BS, i.e.,

$$
\begin{array}{cl}
\max & C=\sum_{i=1}^{K} \sum_{j=1}^{N_{m, i}} \log _{2}\left(1+\frac{\lambda_{i, j}^{2}}{\sigma_{i}^{2}} p_{i, j}\right) \\
\text { s.t. } & \sum_{i=1}^{K} \sum_{j=1}^{N_{m, i}} p_{i, j}=p^{\max } \\
& p_{i, j} \geq 0,1 \leq i \leq K, 1 \leq j \leq N_{m, i}
\end{array}
$$

where $p^{\max }$ is the maximum allowed transmission power at the BS.

As in Problem 1, in order to allocate power to each data stream, it is necessary to use OSDM to decompose the multiuser MIMO system into SISO subchannels. The waterfilling algorithm is then used to solve Problem 2 to maximize the capacity of all users combined. The results of the power allocation may result in not allocating any power for channels with lower SNR. As in problem 1, we need to adjust the structure of the equivalent MIMO system, decompose the new channel using OSDM, and apply the water-filling algorithm again to obtain optimal power allocation iteratively until the powers allocated to all data streams are non-zero. As shown in Fig. 2, the OSDM with global power allocation can be described as follows.

1) Initialize: $\tilde{N}_{m, i}=N_{m, i}(i=1, \ldots, K)$.

2) Calculate $\lambda_{i, j}\left(j=1, \ldots, \tilde{N}_{m, i}\right), W_{i}$ and $T_{i}(i=$ $1, \ldots, K)$ with the iterative OSDM method.

3) Obtain the optimal power allocation $p_{i, j}^{o p t}(i=1, \ldots, K$, $\left.j=1, \ldots, \tilde{N}_{m, i}\right)$ to maximize the capacity of all users combined with a constraint on the total transmit power using the water-filling algorithm, and set $p_{i, j}^{o p t}=0(i=1, \ldots, K$, $\left.j=\tilde{N}_{m, i}+1, \ldots, N_{m, i}\right)$.

4) Iterate steps 2) and 3) until no further reduction on the number of subchannels $\tilde{N}_{m, i}$ for every user is observed.

Similarly, as for the local power allocation, the above iterative process is convergent since $\tilde{N}_{m, i}$, which is used to indicate the end of this process, is finite and monotonically decreased.

\section{Simulation And Results}

The multiuser multistream MIMO system is simulated to evaluate the performance of the proposed schemes in terms of capacity. All simulation results are averaged over 2000 channel realizations to obtain capacity curves. The simulated channel is a flat fading MIMO channel, whose elements are zero mean complex Gaussian random variables with unity variance. The noise variance per antenna is assumed to be the same for all users, $\sigma_{1}^{2}=\ldots=\sigma_{K}^{2}=\sigma^{2}$. To better compare the performance of the two proposed schemes to the original OSDM scheme, the mean total channel capacity and the outage capacity are shown in Fig. 3 and Fig. 4 for different antenna configurations. In these figures, OSDM, OSDMLPA and OSDMGPA denote the ordinary OSDM scheme, OSDM

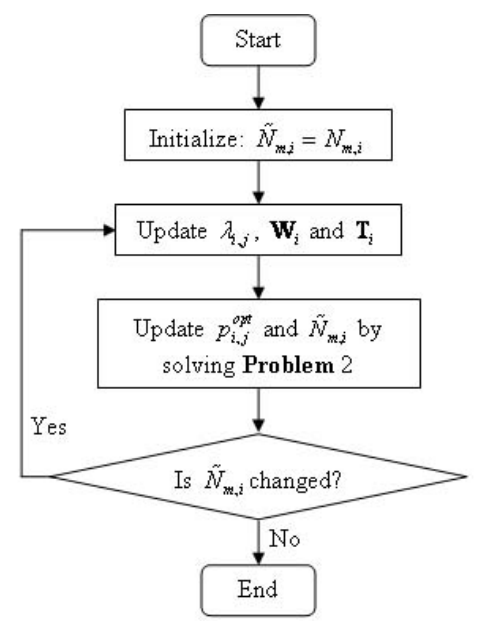

Fig. 2. Flow diagram representation of the global power allocation scheme.

with local power allocation and OSDM with global power allocation, respectively.

Fig. 3 shows the capacity for the following antenna configuration: $N_{t}=9, K=3, N_{m, 1}=4, N_{m, 2}=3, N_{m, 3}=2$, $N_{r, 1}=6, N_{r, 2}=4, N_{r, 3}=2$. The allowed maximal power is $p_{i}^{\max }=3(i=1, \ldots, K)$ and $p^{\max }=9$. In Fig. 4, the antenna configuration and the allowed maximal power are $N_{t}=9$, $K=3, N_{m, i}=3, N_{r, i}=3, p_{i}^{\max }=3(i=1, \ldots, K)$ and $p^{\max }=9$.

From these figures, it is clear that the total capacity of the OSDM with global power allocation is greater than ordinary OSDM and OSDM with local power allocation under the same conditions. As shown in Fig. 3 (b), the probability that total capacity is less than $30 \mathrm{bits} / \mathrm{sec} / \mathrm{Hz}$ is 0.31 for OSDMGPA approach, while it is 0.37 for OSDMLPA and 0.41 for original OSDM. This observation becomes more obvious when SNR is lower, for example, at SNR $=-5 \mathrm{~dB}$ in Fig. 3 (a), the OSDMGPA and OSDMLPA approaches have approximately a total capacity of $15.5 \mathrm{bits} / \mathrm{sec} / \mathrm{Hz}$, whereas the original OSDM scheme only achieves $12.6 \mathrm{bits} / \mathrm{sec} / \mathrm{Hz}$.

The OSDM with local power control scheme allocates fixed power for each user to transmit the data symbols and performs power allocation to multiple data stream separately for each user to maximize individual user capacity. Hence, the sum capacity of this method is less than OSDM with global power control scheme. However, the subchannel performance of an individual user can be separately controlled using OSDM with the local power allocation.

In Fig. 3, different users have different numbers of data streams and receive antennas. For the 3rd user, the number of the data streams and the receive antennas are the same (equal 2). But, for other users, they are different, and the numbers of receive antennas are greater than the numbers of data streams. It means that the subchannel performance of user 3 will be worse than other users, if the power can not be globally allocated. So, the difference of the channel capacities using local and global power allocation in Fig. 3 is more obvious than in Fig. 4, for example, the total capacity 


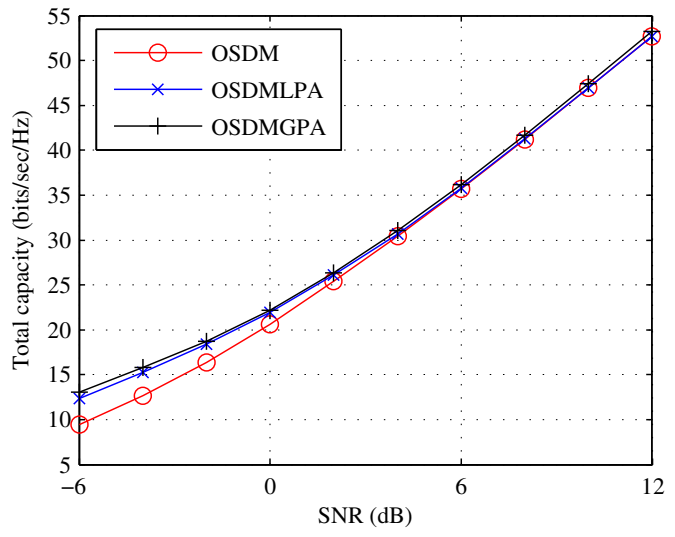

(a) Total channel capacity

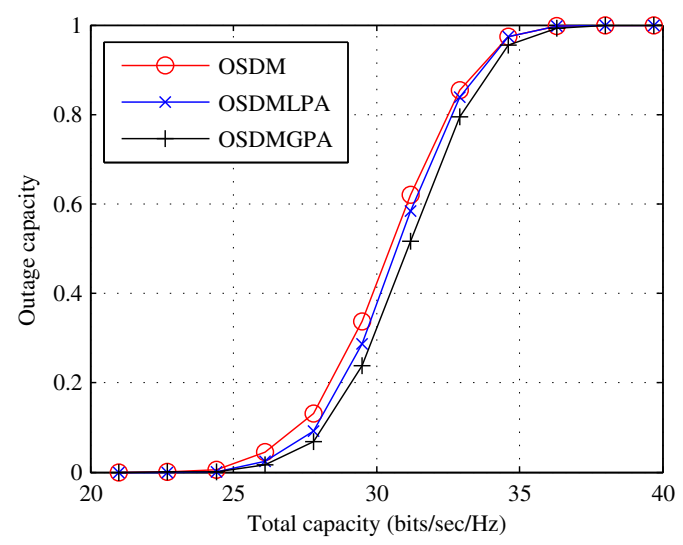

(b) Outage capacity when $\mathrm{SNR}=4 \mathrm{~dB}$

Fig. 3. Capacity comparison for $N_{t}=9$ transmit antennas and $K=3$ users, each equipped with $N_{m, 1}=4, N_{m, 2}=3, N_{m, 3}=2, N_{r, 1}=6, N_{r, 2}=4$ and $N_{r, 3}=2$. The maximal power constraint is $p_{i}^{\max }=3(i=1, \ldots, K)$ and $p^{\max }=9$.

is at least $3 \mathrm{bits} / \mathrm{sec} / \mathrm{Hz}$ greater for the proposed schemes over the entire range of SNR.

\section{CONCLUSION}

In this paper, we designed two kinds of OSDM schemes with power control for the MIMO wireless downlink systems by constraining the maximum total power or the maximum individual powers. The resulting techniques exploit spatial water-filling. Then, the advantages of including the waterfilling algorithm into the OSDM design using local power allocation and global power allocation techniques are shown by simulations. The simulation results indicate that these two proposed schemes yield greater capacity than the original OSDM method. Since the OSDM approach with local power control scheme is able to allocate fixed power for each user to transmit the data symbols and perform power allocation to maximize individual user capacity, it can be used to provide communication services with prescribed quality. When all users have the same priority, the OSDM scheme with global power allocation can be employed within a multiuser wireless communication system.

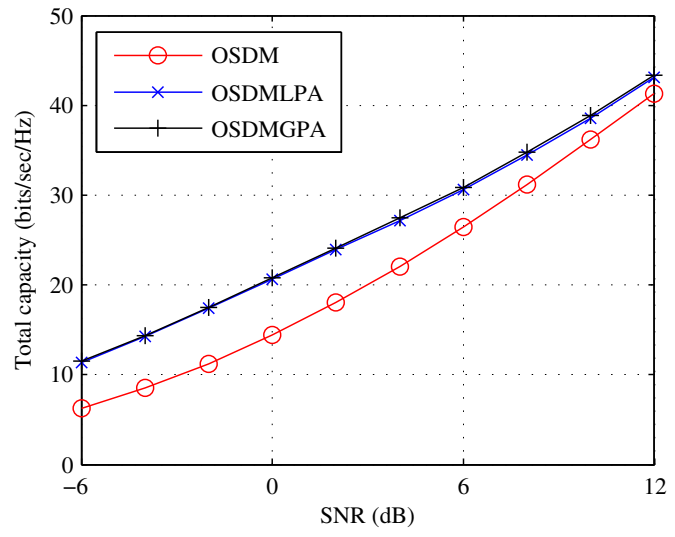

(a) Total channel capacity

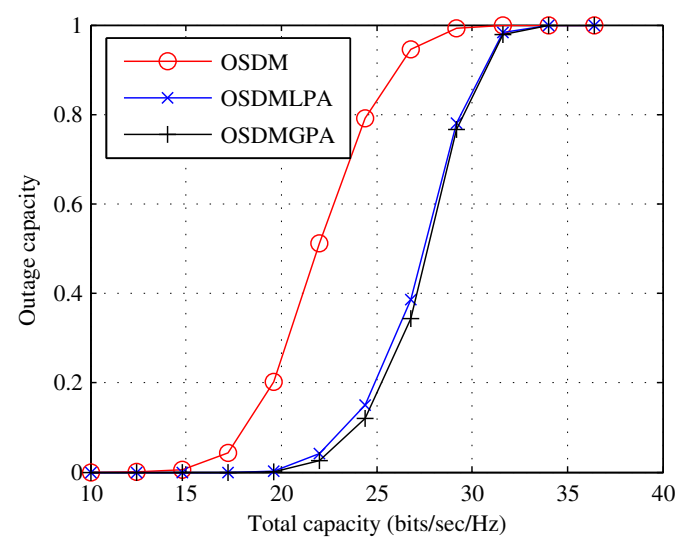

(b) Outage capacity when $\mathrm{SNR}=4 \mathrm{~dB}$

Fig. 4. Capacity comparison for $N_{t}=9$ transmit antennas and $K=3$ users, each equipped with $N_{m, i}=3$ streams and $N_{r, i}=3$ receive antennas $(i=1, \ldots, K)$. The maximal power constraint is $p_{i}^{\text {max }}=3(i=1, \ldots, K)$ and $p^{\max }=9$.

\section{REFERENCES}

[1] G. J. Foschini and M. J. Gans, "On limits of wireless communications in a fading environment when using multiple antennas," Wireless Personal Communications, vol. 6, pp. 311-335, March 1998.

[2] Y. Song and S. D. Blostein, "MIMO channel capacity in co-channel interference," in Proc. 21st Biennial Symposium on Communications, Kingston,Canada, May 2002, pp. 220-224.

[3] Q. H. Spencer, C. B. Peel, A. L. Swindlehurst and M. Haardt, "An introduction to the multi-user MIMO downlink," IEEE Communications Magazine, vol. 42, no. 10, pp. 60-67, October 2004.

[4] K.-K. Wong, R. D. Murch and K. B. Letaief, "A joint-channel diagonalization for multiuser MIMO antenna systems," IEEE Transactions on Wireless Communications, vol. 2, no. 4, pp. 773-786, July 2003.

[5] R. S. Blum, "MIMO capacity with interference," IEEE Journal on Selected areas in communications, vol. 21, no. 5, pp. 793-801, June 2003.

[6] S. Cartreux, L. J. Greenstein, and P. F. Dressen, "Simulation results for an interference-limited multiple-input multiple-output cellular system," IEEE Communications Letters, vol. 4, no. 11, pp. 334-336, November 2000.

[7] J. Liu, Y. T. Hou, Y. Shi, H. D. Sherali and S. Kompella, "On the capacity of multiuser MIMO networks with interference," IEEE Transactions on Wireless Communications, vol. 7, no. 2, pp. 488-494, Feb. 2008.

[8] W. Yu, G. Ginis and J. M. Cioffi, "Distributed multiuser power control for digital subscriber lines," IEEE Journal on Selected Areas in Coтmunications, vol. 20, no. 5, pp. 1105-1115, June 2002.

[9] Z. Pan, K.-K. Wong and T.-S. Ng, "Generalized multiuser orthogonal space-division multiplexing," IEEE Transactions on Wireless Communications, vol. 3, no. 6, pp. 1969-1973, November 2004. 